\title{
The compensational boundary method to calculate the projected contact area of nanoindentation in atomistic simulations
}

\author{
Zhenhai Xu ${ }^{1,2,3}$, Yihui Zhao ${ }^{3}$, Lin Yuan ${ }^{1}$, Yi Qin ${ }^{3}$, Mingjun Chen ${ }^{2}$, Debin Shan ${ }^{1 *}$ \\ ${ }^{1}$ National Key Laboratory for Precision Hot Processing of Metals \& School of Materials Science and Engineering, \\ Harbin Institute of Technology, Harbin 150001, China \\ ${ }^{2}$ School of Mechatronics Engineering, Harbin Institute of Technology, Harbin 150001, China \\ ${ }^{3}$ Centre for Precision Manufacturing, Department of Design, Manufacture and Engineering Management, The \\ University of Strathclyde, Glasgow G1 1XJ, UK
}

*Corresponding authors. E-mail: d.b.shan@gmail.com

\begin{abstract}
The atomistic simulation of nanoidentation has become a powerful method to probe the mechanical behaviour and properties of small volumes of materials. It is crucial to calculate the projected contact area (PCA) accurately in order to obtain a reliable value of nanoindentation hardness. In this work, atomistic simulations of nanoindentation were performed on the $\mathrm{Cu}(111)$ and $\mathrm{Ag}(111)$ surfaces, and a new compensational boundary method is proposed to calculate the PCA. Compared with other available methods, this method provides a clear physical implication, and works well independently of the contact depth and the deformation behaviour of the material. It is also concluded that the widely-used experimental Oliver-Pharr (O-P) method significantly underestimates the PCA in atomistic simulations, and does not work for shallow nanoindentation at the nanoscale.
\end{abstract}

Keywords: Projected contact area; Nanoindentation; Atomistic simulation.

\section{Introduction}

Nanoidentation has become a simple but reliable and powerful experimental technique for probing the mechanical response of small volumes of materials. The increasingly high levels of control, sensitivity, and data acquisition offered by nanoindentation testers have resulted in numerous advances in materials science, particularly regarding the fundamental mechanisms of material behaviour at the nanoscale and even atomic scale. Integrated with other techniques, such as atomic force microscopy (AFM), electron imaging, diffraction, heating and electrical contact resistance measurement, nanoindentation experiments can be performed in situ to study the interplay between mechanical, thermal and electrical effects of materials $[1,2]$. At the nanoscale, the atomistic simulation, which tracks atoms' motion mainly based on the Newton's second law (for molecular dynamics) or energy minimization (for molecular statics), is another well-established technique to study material behaviour from the atomic view. It has been very helpful to reveal the atomic processes underlying the nanoindentation response of materials $[3,4]$.

Conventional hardness is defined as the test load divided by the surface area or projected surface area of the residual impression after the removal of the load, which is often measured with naked eyes or with optical microscopes, while the nanoindentation hardness is defined as the test load divided by the projected area of the contact between the tested material and the indenter at that load, which area is

This is a pre-copyedited, author-produced PDF of an article accepted for publication in Computational Materials Science (2016, 112: 185-192) following peer review. The formal publication is available at Elsevier via http://dx.doi.org/10.1016/j.commatsci.2015.10.026.

(C) 2015. This manuscript version is made available under the CC-BY-NC-ND 4.0 license $\mathrm{http}: / /$ creativecommons.org/licenses/by-nc-nd/4.0/ 
too small to be measured optically. How to acquire the PCA accurately has always been a critical issue in nanoindentation experiments and simulations. The PCA is often defined as an area function of the contact depth for a specific shaped indenter. The O-P method has been adopted widely to determine the area function and contact depth, and undergone numerous refinements and changes due to the advances of testing techniques and understanding of the contact mechanics. In this method, the area function is validated by using materials of known Young's modulus, and the contact depth is calculated from an analysis of the load-depth curve based on Hertzian contact theory [5,6]. The AFM can be used to directly measure the area function, rather than the actual contact area due to the elastic recovery of tested materials during withdrawal of the indenter [7, 8]. Additional corrections must be made for the evaluation of the PCA due to the imperfect indenter geometry, the tilt and surface defects of samples, and the pile-up/sink-in behaviour of tested materials [9-13].

The O-P scheme was applied to calculate the PCA in atomistic simulations of nanoindentation by $\mathrm{Ju}$ et al [14], resulting in a higher hardness for $\mathrm{Cu}(001), \mathrm{Cu}(110)$ and $\mathrm{Cu}(111)$ surfaces than the experimental values. It can be accounted by the result of Jeng and Tan's atomistic simulation of nanoindentation on the $\mathrm{Cu}(001)$ surface that $\mathrm{O}-\mathrm{P}$ method underestimates the PCA [15]. However, the inability of the $\mathrm{O}-\mathrm{P}$ method needs to be studied further by nanoindentation simulations along other crystal orientations due to the anisotropy of metal crystals $[16,17]$. On the other hand, each atom in the sample and the indenter can be easily located in atomistic simulations of nanoindentation, which make it feasible to acquire the PCA directly from the atomic configuration of the sample under the indenter or that of the indenter. Two steps are usually taken to achieve this goal: (i) distinguishing the effective atoms contributing to the PCA from the others by some criterion, and (ii) calculating the PCA by a proper method based on these effective atoms. Before applying this idea, two types of indenter models should be understood: the physical one consisting of atoms and the virtual spherical one without any atomic detail. The interactions between indenters and samples are described by a semi-empirical manybody or pair potential $[14,15,18,19]$, and a simple empirical repulsive pair function[4, 17, 20, 21]. If the atoms in the sample and/or the indenter undergo an exterior repulsive force, they are identified as the effective contact atoms. Chen and Ke [18] presented two approaches to calculate the PCA based on the projections of the effective contact atoms in the physical indenter identified by the repulsive force: (i) the circular approximation of the projections' boundary considering the action range of indenter atoms, and (ii) the polygonal approximation by connecting all contact atoms. However, they cannot be applied directly to the atomistic simulations using the virtual indenter due to no atomic detail being available for the indenter. Lilleodden et al [21] took the area of the polygon with vertices at the projections of contact atoms in the sample as the PCA. Jeng and Tan [15] used the contact pressure to identify the PCA. These polygonal approximation algorithms ignore the contribution from the partial area outside the boundary occupied by atoms in the polygonal boundary. The problem will become serious in the case of a small number of contact atoms when the depth is shallow.

In order to solve these problems, in this paper a new compensational boundary method for the PCA calculation in atomistic simulations is proposed and compared with several other methods to prove its validity. The applicability of the $\mathrm{O}-\mathrm{P}$ method at the nanoscale is also tested by the nanoindentaton simulations on $\mathrm{Cu}(111)$ and $\mathrm{Ag}(111)$ surfaces.

\section{Computational details}

The nanoindentation was performed on the (111) surface of a single crystal with dimensions of $13[11 \overline{2}] a \times 23[110] a \times 10[111] a$ as shown in Fig. 1a, where $a$ is the lattice constant, $3.615 \AA$ for $\mathrm{Cu}$ and $4.09 \AA$ for Ag. The embedded atom method (EAM) potentials developed by Mishin et al $[22,23]$ were adopted to model the atomic interaction. The top surface of the crystal is traction free, while the bottom three layers of atoms are fixed and the periodic boundary conditions are applied to the other surfaces. The virtual spherical indenter was adopted and represented by a repulsive potential of the form: 


$$
U=\sum_{i=1, n} U_{i}\left(r_{i}\right), U_{i}\left(r_{i}\right)=\left\{\begin{array}{cc}
k\left(R-r_{i}\right)^{3} / 3, & r_{i}<R \\
0, & r_{i} \geq R
\end{array}\right.
$$

where $n$ is the number of atoms in the sample, $k$ is a force constant, $R$ is the indenter radius, and $r_{i}$ is the distance from atom $i$ to the spherical centre of the indenter. Here, they are $k=200 \mathrm{eV} / \AA^{3}$ and $R=40 \AA$ for both simulations on $\mathrm{Cu}$ and $\mathrm{Ag}$. The test load $P$ is defined as the summation of vertical components of the forces $F$ calculated from Eq. (1). If an atom penetrates the boundary of the indenter (i.e., when $r_{i}$ $<R$ ), it will become a contact atom and contribute to $P$ as shown in Fig. $1 \mathrm{~b}$. The number of contact atoms is noted as $n_{\mathrm{c}}$, and the penetration depth of contact atom $i$ is defined as:

$$
h_{\mathrm{pen}}^{i}=R-r_{i}
$$

The indentation was conducted in a displacement-control mode by initially positioning the indenter above one of the free surface atoms, which is named as the first contact atom for convenience of reference. The loading process of nanoindentation commences after the indenter contacts the first contact atom. The indentation depth $H$ is defined as:

$$
H=\Delta H\left(n_{\text {step }}^{\text {load }}-n_{\text {step }}^{\text {unload }}\right)
$$

where $\Delta H$ is the displacement of the indenter at each step, here $\Delta H=0.1 \AA, n_{\text {step }}^{\text {load }}$ is the step number of loading, and $n_{\text {step }}^{\text {unload }}$ is the step number of unloading before $P$ becomes zero. The depths at which the unloading process commences and ends are noted as $H_{\max }$ and $H_{\mathrm{p}}$, respectively. At each step the equalized atomic configuration was found using energy minimization by the open code LAMMPS [24]. The load-depth curves of nanoindentation were plotted from the variables of $P$ and $H$.

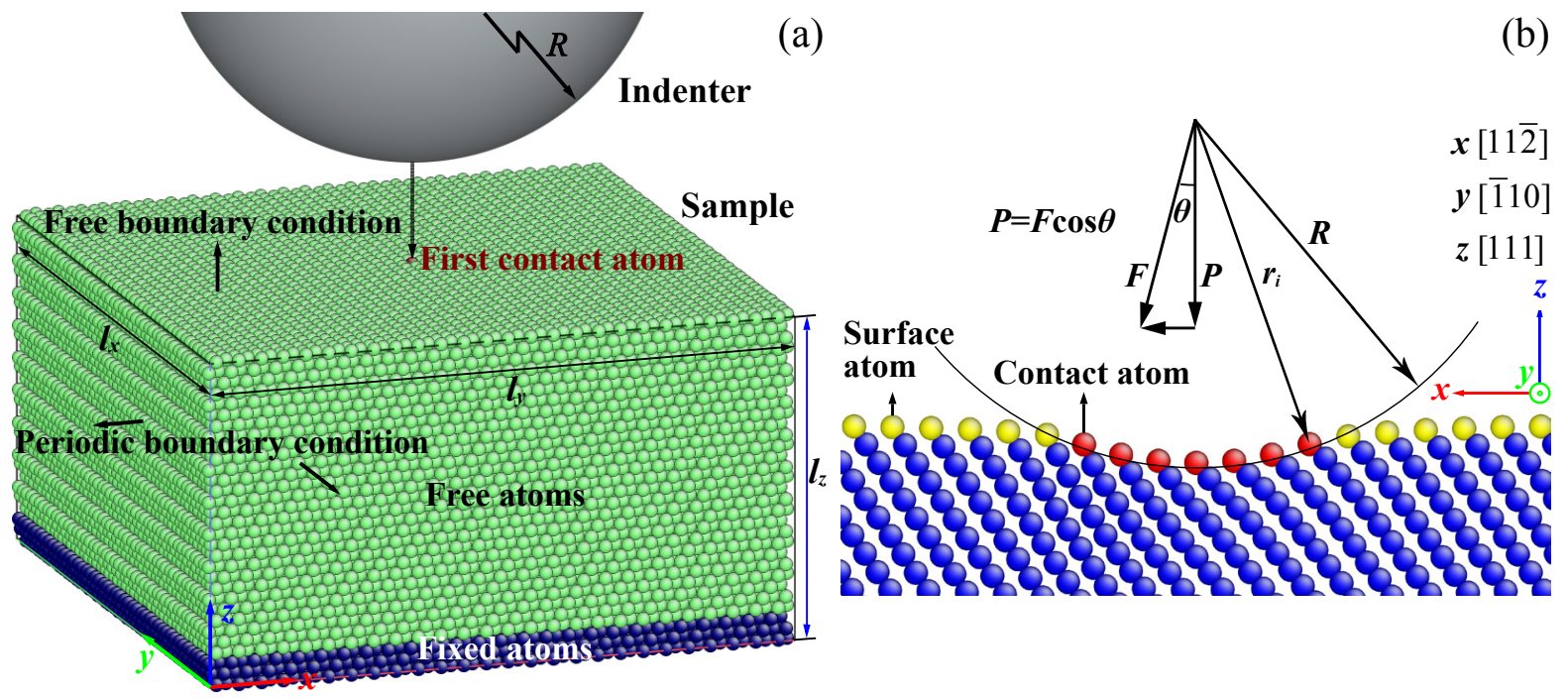

Fig. 1. Atomistic model of nanoindentation on the (111) surface: (a) three-dimensional (3D) one, and (b) $2 \mathrm{D}$ one on a single (110) atomic plane.

\section{Results and discussion}

\subsection{Methods to calculate the PCA in atomistic simulations}

The sample underwent an elastic deformation before the emission of the initial dislocations during nanoindentation. The atoms in the deformed sample surface are same with ones in the initial sample surface, and can be easily identified. Fig. 2a show the surface atoms around the indenter and their projections on the plane perpendicular to the loading direction at $H=0.6 \AA$. The projections of contact atoms are surrounded by a black circle, and the area occupied by them is the PCA, which can be calculated by two methods as shown in Fig. 2b-c. Each surface atom and its projection in Fig. 2a-b are 
coloured by its distance from the initial position of the first contact atom along the loading direction $h_{z}^{i}$, while each contact atom projection in Fig. $2 \mathrm{c}$ is coloured by its penetration depth $h_{\mathrm{pen}}^{i}$. The white point encircled by a black boundary marks the projection of the indenter centre.

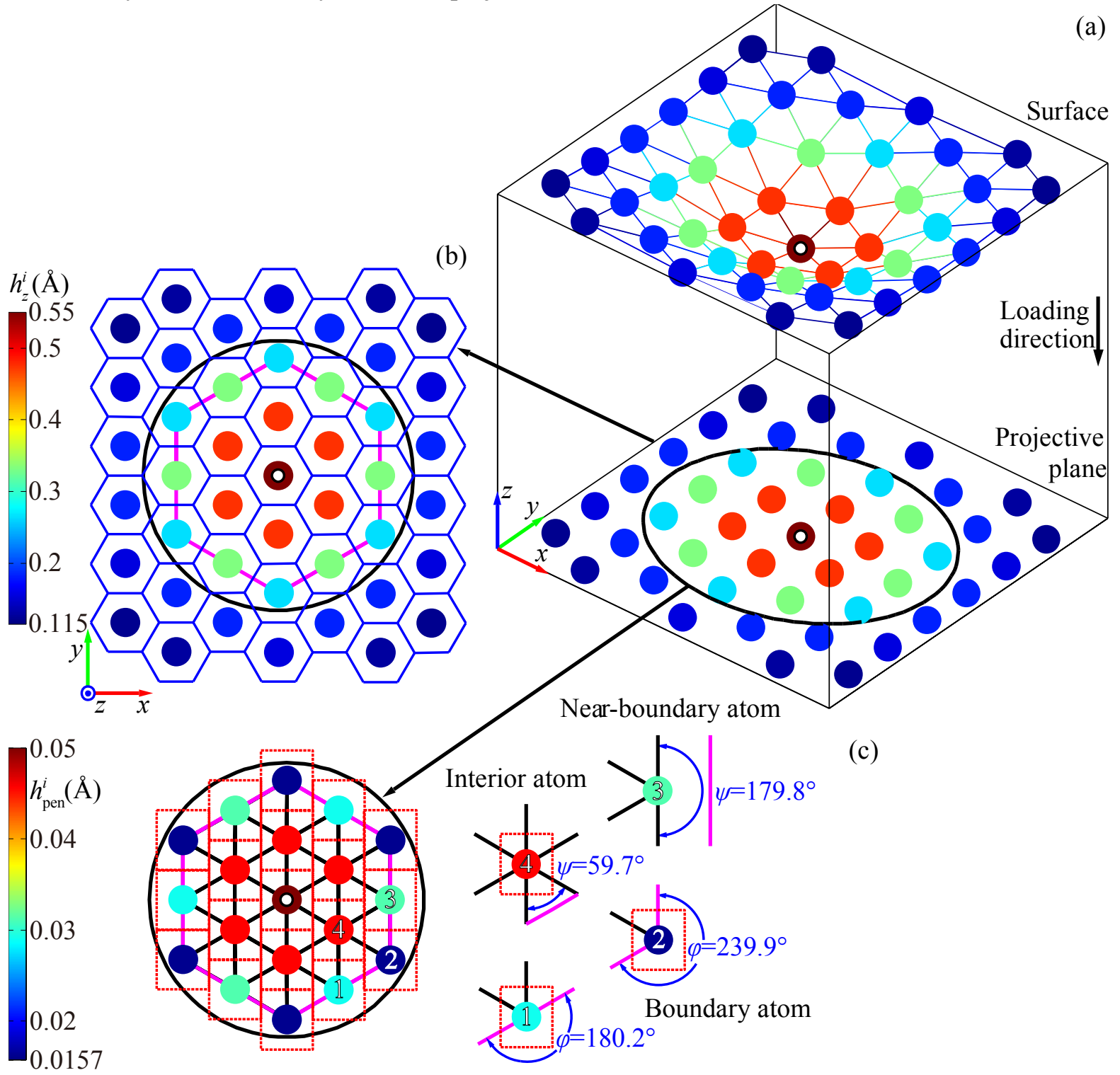

Fig. 2. Partition of the projection plane of the surface atoms around the indenter by two methods to calculate the PCA at $H=0.6 \AA$ : (a) surface atoms and their projections on the plane perpendicular to the loading direction, and which is partitioned by (b) the Voronoi diagram method and (c) the compensational boundary method.

\subsubsection{Voronoi diagram method}

As shown in Fig. 2b, the projection plane is partitioned into a Voronoi diagram [25], which makes each surface atom projection occupy a convex polygon consisting of all points closer to that projection than to any other. Therefore, the Voronoi polygon of each atom projection encircles the region actually occupied by that projection, and the area sum of the Voronoi polygons of the contact atom projections is the true physical PCA, noted as $A_{\mathrm{c}}^{\mathrm{Vor}}$. As a common crystal defect, the surface step forms when dislocations emit to account for the plastic deformation, resulting in the appearance of non-contact surface atoms inside the boundary polygon connected by the adjacent contact atom projections. Fig. 3a shows an example of this case at $H=4.2 \AA$, where the contact atom projections are marked by white 
solid circles, and the non-contact atom projections without marks are located at the vicinity of the vertices of the red triangle. The areas of these non-contact atom projections should be taken into account in the calculation of the PCA. However, with the aggravation of the plastic deformation during nanoindentation as shown in Fig. 3c, there is no simple and effective criterion to distinguish surface atoms from interior atoms due to the complex surface morphology, resulting in the Voronoi diagram of the set of surface atom projections being unable to be worked out. Therefore, the Voronoi diagram method is applicable to calculate the PCA only before the emission of the initial dislocations.
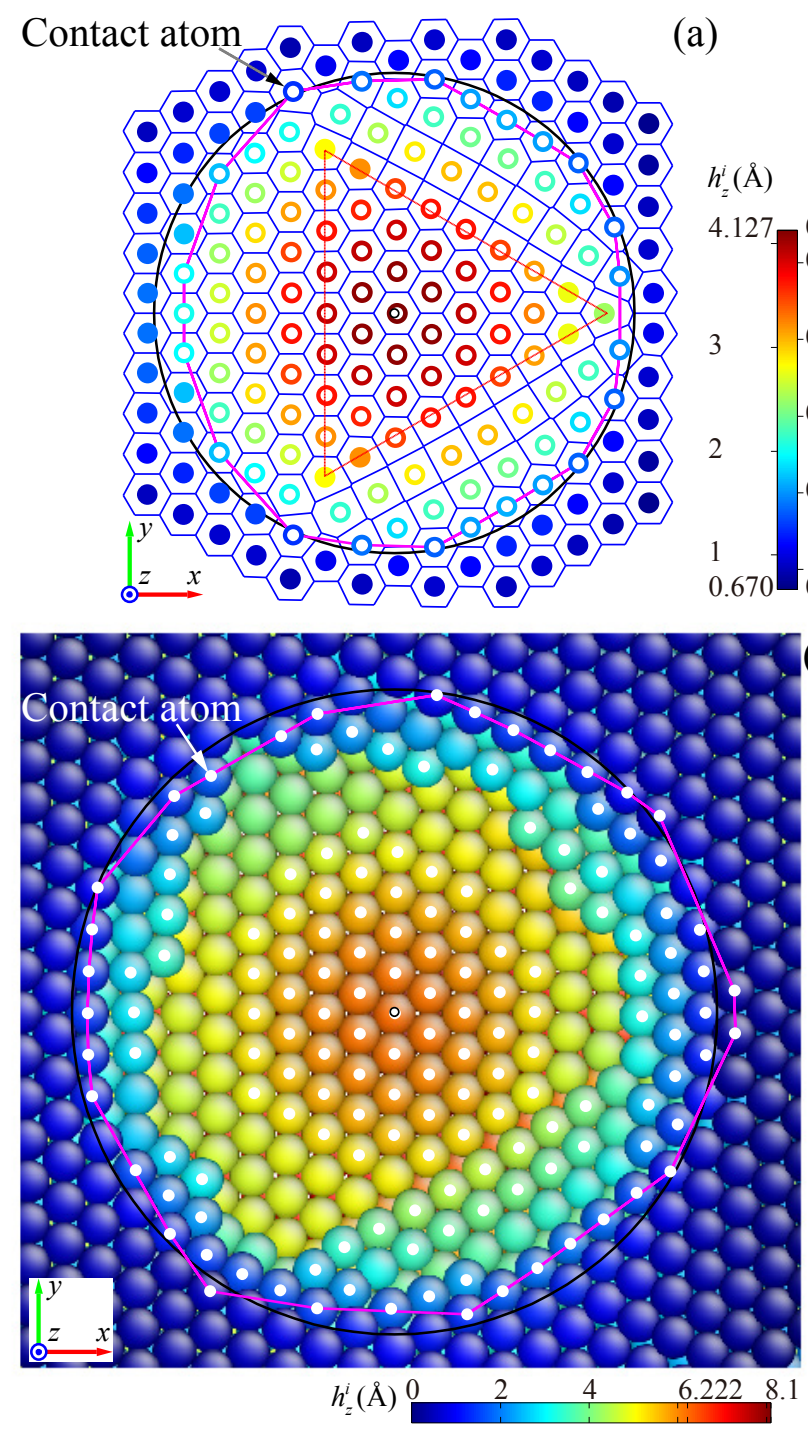

(a)

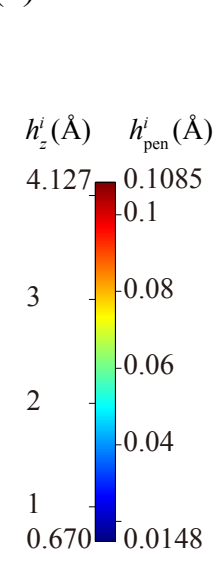

(b)

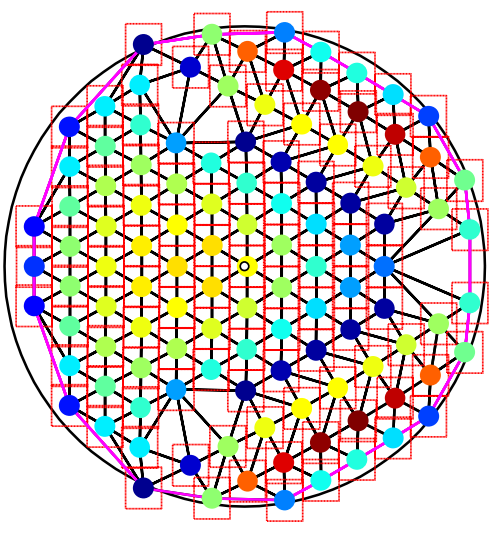

(d)

Fig. 3 Surface atom projections and their Voronoi diagram and Delaunay triangulation: (a) surface atom projections and their Voronoi diagram, and (b) contact atom projections and their Delaunay triangulation with the convex hull at $H=4.2 \AA$; (c) the top view of the atomic configuration of the sample surface, and (d) contact atom projections and their Delaunay triangulation with the convex hull at $H=6.3$ $\AA$.

\subsubsection{Compensational boundary method}

During the whole process of nanoindentation the contact atoms can always be identified easily by finding the atoms in the sample subjected to the repulsive force from the indenter, and their projections can be used to define the boundary of the projected contact surface, resulting in a feasible calculation of the main part of the PCA based on this boundary. Then, the areas of the boundary atom projections outside the boundary are used to compensate the main part to finalize the PCA. This is the basic idea of 
the proposed compensational boundary method to calculate the PCA. The key issues of this method include the constitution of the boundary of the projected contact surface, identification of the compensational atom projections and the compensation solution. Here, a simple and easy algorithm based on the Matlab ${ }^{\circledR}$ functions is given as an example of realization. The convex hull [25] of the set of contact atom projections, which is the smallest convex set containing all of these projections, is defined as the boundary coloured in pink as shown in Figs. $2 \mathrm{c}$ and 3, and its area is noted as $A$ hul. Both of the atom projections in the boundary and close to the boundary contribute to the compensation item of the PCA. In order to identify the near-boundary atom projections the Delaunay triangulation [25] of the convex hull, which is the dual graph of the Voronoi diagram, is introduced. This type of the compensational boundary method is performed as given in the following steps:

(I) The initial indented surface area of the sample is divided by the number of surface atoms, and the average is noted as $A$ rec. Assume that each contact atom projection is encircled by a rectangle with an area of $A_{\text {rec }}$ as shown in Figs. 1c, $3 \mathrm{~b}$ and $3 \mathrm{~d}$.

(II) The exterior angle of the boundary polygon, for example, those at the vertices 1 and 2 in Fig. $2 \mathrm{c}$, is noted as $\varphi$.

(III) The angle opposite to the edge of the boundary polygon is noted as $\psi$. Here, the atom projection with $\psi>175^{\circ}$ is defined as the near-boundary atom projection, for example, the atom projection 3 in Fig. 2c.

(IV) The compensational area from both the boundary atom projections and near-boundary atom projections can be calculated as:

$$
A_{\mathrm{com}}= \begin{cases}A_{\mathrm{rec}} \cdot n_{\mathrm{c}}, & n_{\mathrm{c}}<3 \\ A_{\mathrm{rec}}\left(\frac{\varphi}{360} \cdot n_{\varphi}+\frac{\psi}{360} \cdot n_{\psi}\right), & n_{\mathrm{c}} \geq 3\end{cases}
$$

where $n_{\varphi}$ and $n_{\psi}$ are the numbers of the boundary atom projections and near-boundary atom projections, respectively.

(V) The PCA is calculated as:

$$
A_{\mathrm{c}}^{\mathrm{bou}}= \begin{cases}A_{\mathrm{com}}, & n_{\mathrm{c}}<3 \\ A_{\text {hul }}+A_{\mathrm{com}}, & n_{\mathrm{c}} \geq 3\end{cases}
$$

Based on the value of $A_{\mathrm{c}}^{\text {bou }}$, the contact radius can be defined as:

$$
a_{\mathrm{c}}=\sqrt{A_{\mathrm{c}}^{\mathrm{bou}} / \pi}
$$

The black hollow circles with a radius of $a_{\mathrm{c}}$ centred at the projection of the indenter centre are plotted in Figs. 2 and 3. It can be seen that the circle occupies nearly the same area with that calculated by the Voronoi diagram method. The Matlab ${ }^{\circledR}$ script for this algorithm is available via email to the authors".

It should be noted that in order to save the computational time, the atomistic simulations of nanoindentation in this research were performed by the virtual spherical indenter and the energy minimization method; however, the compensational boundary method is applicable to calculate the PCA for other atomistic simulations of nanoindentation, no matter what type of indenter is (physical or virtual), what their shapes are, nor the simulation methods adopted (energy minimization or molecular dynamics). The energy minimization and molecular dynamics apply different methods to track atoms' motion within the simulation system, but the resulted atomic configurations, which are used to calculate the PCA, are the same. The nanoindentation simulations using any shaped virtual indenter and physical indenter follow the same basic principle to identify the contact atoms, while the specific steps are different. For the virtual indenter, if the distance between the sample atom and the indenter falls in the distance region of repulsive force, the atom is identified as a contact atom. For the physical indenter, if an atom in the sample undergoes a repulsive force from the indenter, it will be taken as a contact atom.

\footnotetext{
*Email: zhenhai.xu8@gmail.com.
} 
Compared with the simulation using the virtual spherical indenter, more computational steps and time are needed to identify the contact atoms for the simulation using the physical and/or other shaped indenter. Once the contact atoms are identified from the atomic configuration of the sample, the proposed method could be adopted and carried out in the same way as above.

\subsection{Validity of the compensational boundary method}

\subsubsection{Comparison with other numerical methods in atomistic simulations}

To check the reliability of the compensational boundary method, except the above Voronoi diagram method, two other available methods in atomistic simulations are also introduced to calculate the PCA for the nanoindentation of $\mathrm{Cu}(111)$ to different indentation depths. One method is based on the constant rectangle area $A_{\text {rec }}$ of contact atoms, and defined as:

$$
A_{\mathrm{c}}^{\mathrm{rec}}=A_{\mathrm{rec}} \cdot n_{\mathrm{c}}
$$

The other method is based on the Hertzian contact theory used at the macroscale, and depends on the contact depth. It is defined as:

$$
\begin{gathered}
A_{\mathrm{c}}^{\text {Her }}=\pi\left(a_{\mathrm{c}}^{\text {Her }}\right)^{2} \\
a_{\mathrm{c}}^{\text {Her }}=\sqrt{R^{2}-\left(R-h_{\mathrm{c}}\right)^{2}}=\sqrt{2 R h_{\mathrm{c}}-h_{\mathrm{c}}^{2}}=\sqrt{R h_{\mathrm{t}}-h_{\mathrm{t}}^{2} / 4}
\end{gathered}
$$

where $h_{\mathrm{c}}$ and $h_{\mathrm{t}}$ are the contact depth and indentation depth as shown in Fig. 4, and $h_{\mathrm{c}}=h_{\mathrm{t}} / 2$.

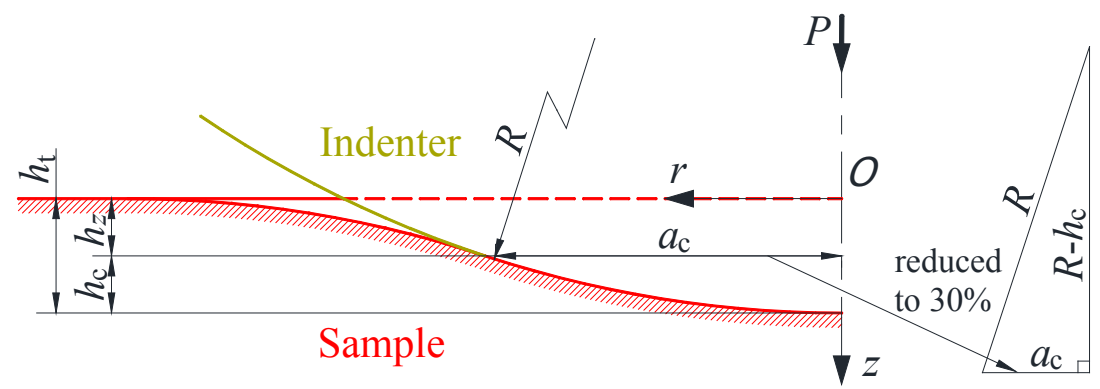

Fig. 4. Schematic of the contact between a rigid spherical indenter and a flat non-rigid specimen.

Before the emission of the initial dislocations at $H=4.1 \AA$, all four methods are feasible to calculate the PCA, their comparison being shown in Fig. 5:

(I) With the increase of $H$, the contact atoms undergo a more severe compression by surrounding atoms, and then the average of each contact atom's Voronoi polygon area becomes smaller. $A_{\text {rec }}$ is constant, and the counting number is the same, resulting in the monotone increase of $A_{\mathrm{c}}^{\text {rec }} / A_{\mathrm{c}}^{\mathrm{Vor}}$ with an average of $1.025 \pm 0.012$ which indicates that $A_{\mathrm{c}}^{\mathrm{Vor}}$ can be roughly approximated by $A_{\mathrm{c}}^{\mathrm{rec}}$.

(II) Due to the relationship of $h_{\mathrm{t}} \ll R$ in the initial stage of nanoindentation, the item $h_{\mathrm{t}}^{2} / 4$ in Eq. (9) approximates zero, and then Eq. (8) is reduced to $A_{\mathrm{c}}^{\text {Her }}=\pi R h_{\mathrm{t}}$. Here, $h_{\mathrm{t}}$ is substituted by $H$, resulting in a near-linear relationship between $A_{\mathrm{c}}^{\text {Her }}$ and $H$ indicated as the green curve. $A_{\mathrm{c}}^{\text {Her }}$ is less than the values calculated by the other three methods except at $H=0.1 \AA$, and the average of $A_{\mathrm{c}}^{\text {Her }} / A_{\mathrm{c}}^{\text {Vor }}$ is $0.798 \pm 0.064$ at $H=0.2-4.1 \AA$, indicating a large deviation of $A_{\mathrm{c}}^{\text {Her }}$ from the true physical PCA. The reason is that the relationship of $h_{\mathrm{t}} / h_{\mathrm{c}}=2$ in the Hertzian contact theory fails in atomistic simulations as show in the top-left inset of Fig. 5, where $h_{\mathrm{t}}$ is the maximum of $h_{z}^{i}$, and $h_{\mathrm{c}}$ is the average distance along the loading direction of the contact atoms far from the first contact atom relative to the deepest contact atom. Here, the contact atoms far from the first contact atom are identified by their projection with a distance of no less $0.1 \AA$ than the distance between the furthest contact atom projection from the first 
contact atom projection. $h_{\mathrm{t}} / h_{\mathrm{c}}$ is always less than the Hertzian value of 2 , resulting in a smaller value of $A_{\mathrm{c}}^{\text {Her }}$ according to Eqs. (8) and (9).

(III) The average and maximum of $A_{\mathrm{c}}^{\text {bou }} / A_{\mathrm{c}}^{\mathrm{Vor}}$ are $0.995 \pm 0.016$ and 0.954 , respectively, i.e., they are very close to each other. Therefore, the assumption of the convex hull combined with the boundary compensation is a reasonable approximation of the true physical PCA. The discrete evolution of $A_{\mathrm{c}}^{\text {bou }}$ results from the discrete nature of the contact region, as shown in Fig. 6. $A_{\mathrm{c}}^{\text {bou }}$ increases with the increase of $n_{\mathrm{c}}$ from 13 at $H=0.5 \AA$ to 19 at $H=0.6 \AA$. With further indentation until $H=0.8 \AA, h_{\text {pen }}^{i}$ increases, but $n_{\mathrm{c}}$ remains constant, resulting in the negligible change in $A_{\mathrm{c}}^{\text {bou }}$.

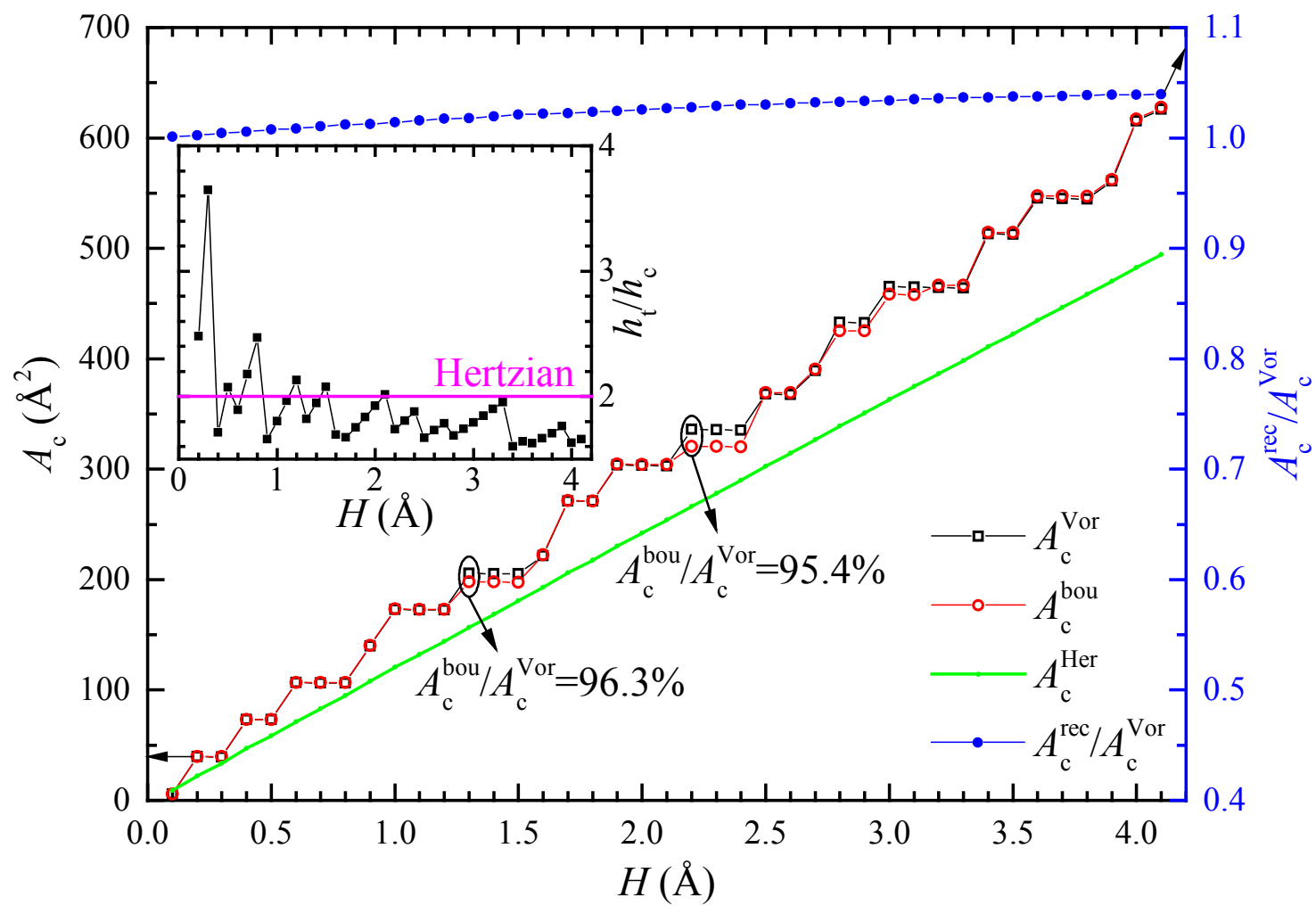

Fig. 5. Comparison of the PCA calculated using different methods before the emission of the initial dislocations.

(a)

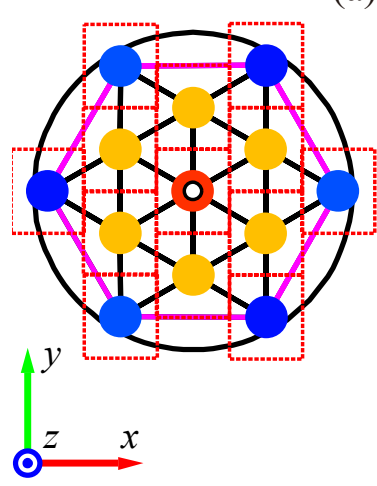

(b)

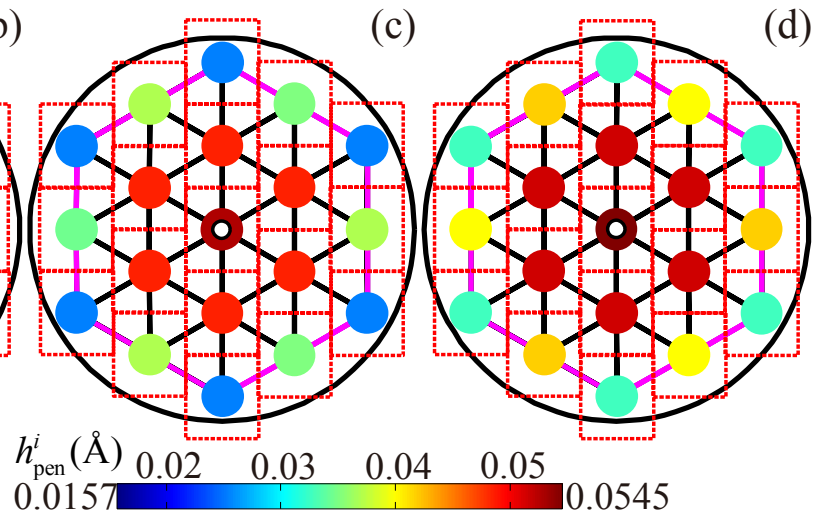

Fig. 6. The discrete evolution of $A_{\mathrm{c}}^{\text {bou }}$ during nanoindentation: (a) $H=0.5 \AA$, (b) $H=0.6 \AA$, (c) $H=0.7 \AA$, (d) $H=0.8 \AA$. 
After the emission of the initial dislocations at $H=4.2 \AA$, only the compensational boundary method is feasible to calculate the value of the PCA. Fig. 7 shows that $A_{\mathrm{c}}^{\text {bou }}$ increases with the increase of $H$. Less plateaux appear in the curve compared with the case before the emission of the initial dislocations, due to the larger area increment for the same loading step with the deeper indentation, as shown in the top-right inset of Fig. 7. The contribution of the boundary compensation and the assessment of its necessity can be characterized by $A_{\text {com }} / A_{\mathrm{c}}^{\text {bou }}$ :

(I) At the initial stage of nanoindentation, the number ratio $\eta$ of the boundary atom projections and near-boundary atom projections with the contact atom projections is high, therefore the boundary compensation contributes greatly to the PCA, resulting in a high value of $A_{\text {com }} / A_{\mathrm{c}}^{\text {bou }}$.

(II) Before the emission of the initial dislocations, $A_{\text {com }} / A_{\mathrm{c}}^{\text {bou }}$ gradually decreases with fluctuation with the increase of $H$, and reaches a minimum of $10 \%$ at $H=3.9 \AA$. The contribution to the PCA from the boundary compensation is significant, and it is necessary to include the boundary compensation in the calculation of the PCA. After the emission of the initial dislocations, $A_{\text {com }} / A_{\mathrm{c}}^{\text {bou }}$ decreases with weaker fluctuation, and reaches a minimum of $3 \%$ at $H=9.8 \AA$. The contribution to the PCA from the boundary compensation becomes weak. Nevertheless, the boundary compensation is acceptable for the deeper indentation, due to its small proportion in the total computational cost in the compensational boundary method.

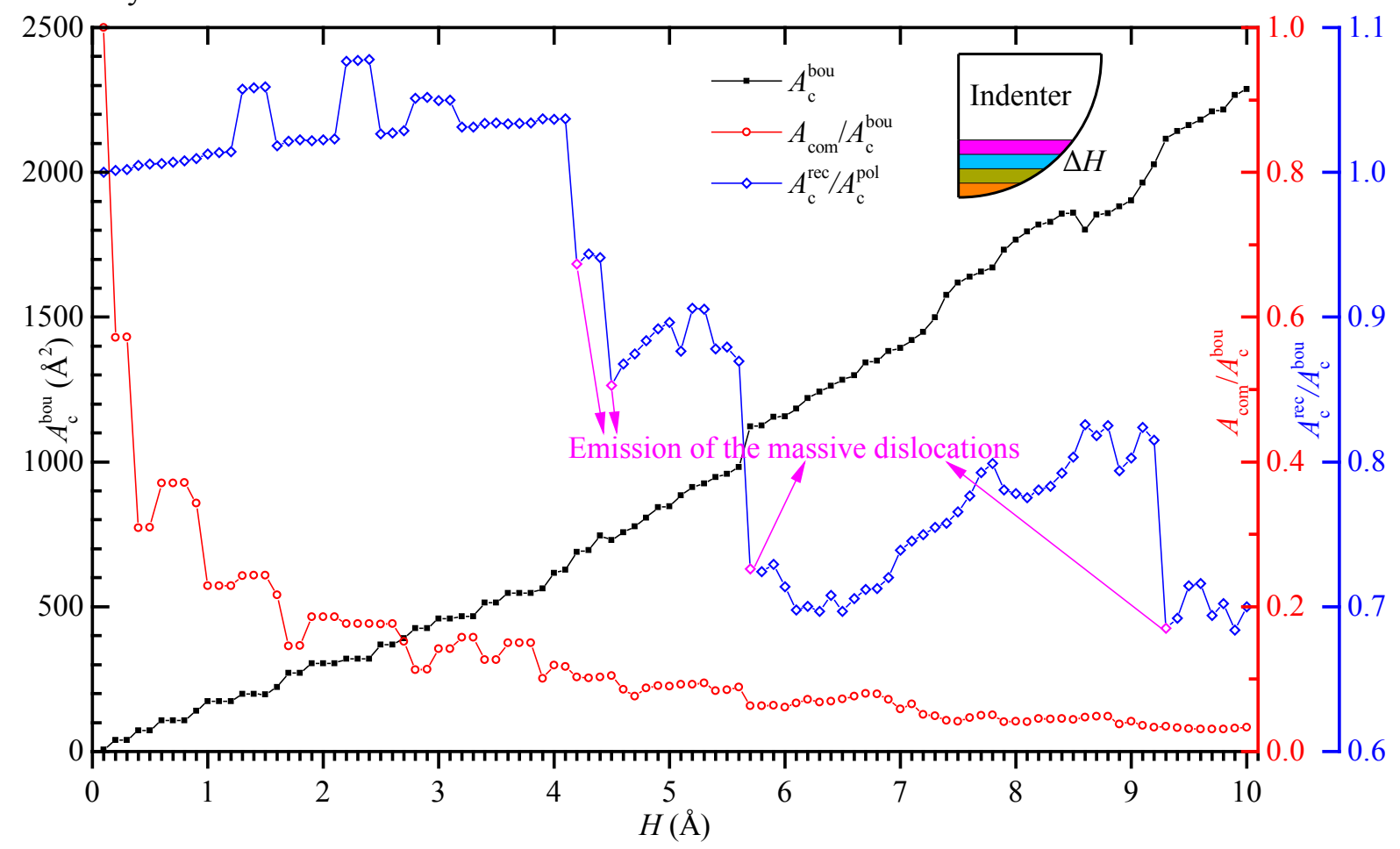

Fig. 7. Variations of $A_{\mathrm{c}}^{\text {bou }}, A_{\text {com }} / A_{\mathrm{c}}^{\text {bou }}$ and $A_{\mathrm{c}}^{\text {rec }} / A_{\mathrm{c}}^{\text {bou }}$ with $H$

$A_{\mathrm{c}}^{\text {rec }} / A_{\mathrm{c}}^{\text {bou }}$ can qualitatively characterize the number ratio of contact atoms and the surface atoms under the indenter. Apparently, the ratio decreases roughly with the depth increase, as shown in Fig. 7. Especially, a sharp drop appears when surface steps form, resulting from the emission of the massive dislocations.

In conclusion, the Voronoi diagram method provides a clear physical implication, and the area calculated by it is a true physical PCA. However, it cannot be applicable to calculate the PCA only after the emission of the initial dislocations due to the failure in identifying surface atoms. By comparison with the Voronoi diagram method, it is established that the compensational boundary method is a 
reasonable approximation of the true physical PCA, and it works well independently of the deformation behavior of the material.

\subsubsection{Comparison with the $O-P$ method}

Compared with the O-P method used widely in experiments, the calculation of the PCA by the compensational boundary method based on contact atoms does not involve the preliminary assessment of the contact depth $h_{\mathrm{c}}$ and the pre-assumption of a specific indentation deformation mode (i.e., pile-up or sink-in). Therefore, $A_{\mathrm{c}}^{\text {bou }}$ is the true physical PCA, resulting in a true and accurate nanoindentation hardness $H_{\mathrm{IT}}^{\text {bou }}$ based on it, which will be compared with the value $H_{\mathrm{TT}}^{\mathrm{O}-\mathrm{P}}$ based on the O-P method.

In order to determine the PCA by the O-P method, the upper $50 \%-80 \%$ of the unloading curve of the test load is taken for the least-square fitting to a simple law relationship:

$$
P=\alpha\left(h-h_{\mathrm{f}}\right)^{m}
$$

where $\alpha, h_{\mathrm{f}}$ and $m$ are constants. A tangent is constructed by differentiating the unloading curve and evaluating at the maximum applied force. The intercept of this tangent with the depth axis yields a depth $h_{\mathrm{r}}$, which determines the contact depth $h_{\mathrm{c}}$ for the spherical indenter together with the maximum depth $h_{\text {max }}$ as:

$$
h_{\mathrm{c}}=0.25 h_{\max }+0.75 h_{\mathrm{r}}
$$

Therefore, the PCA can be calculated by:

$$
A_{\mathrm{c}}^{\mathrm{O}-\mathrm{P}}=\sqrt{2 R h_{\mathrm{c}}-h_{\mathrm{c}}^{2}},
$$

The maximum of $h_{\mathrm{pen}}^{i}$ at $H=6.3 \AA$ is $0.1127 \AA$, as shown in Fig. 3d, but the maximum average of $h_{\mathrm{pen}}^{i}$ during the unloading process is only $0.0673 \AA$, resulting in the rationality of using the indentation depth $H$ for the above fitting procedure in atomistic simulations. The solid lines and scatter plots in Fig. 8 correspond to the loading and unloading processes from $H_{\max }=5,6,7,8,9$, and $10 \AA$ for nanoindentations on $\mathrm{Cu}(111)$ and $\mathrm{Ag}(111)$, respectively. Each sharp drop or increase of the load indicates dislocations emission or annihilation. The initial part of each unloading curve is fitted to Eq. (10), the result being shown as the dashed line. The upper limit of the fitting interval is set at the beginning of the unloading, while the lower limit is taken by these rules: (I) the point before the first sharp change of the slope of the unloading plot (e.g., the point before the first load increase); (II) the point before the second sharp change of the slope of the unloading plot when the first change of the slope is weak and there are only several data points before it; (III) the point at $H=0.8\left(H_{\max }-H_{\mathrm{p}}\right)$ when the unloading plot is smooth. The unloading plots with $H_{\max }=5,8$, and $6 \AA$ follow the three rules, respectively.
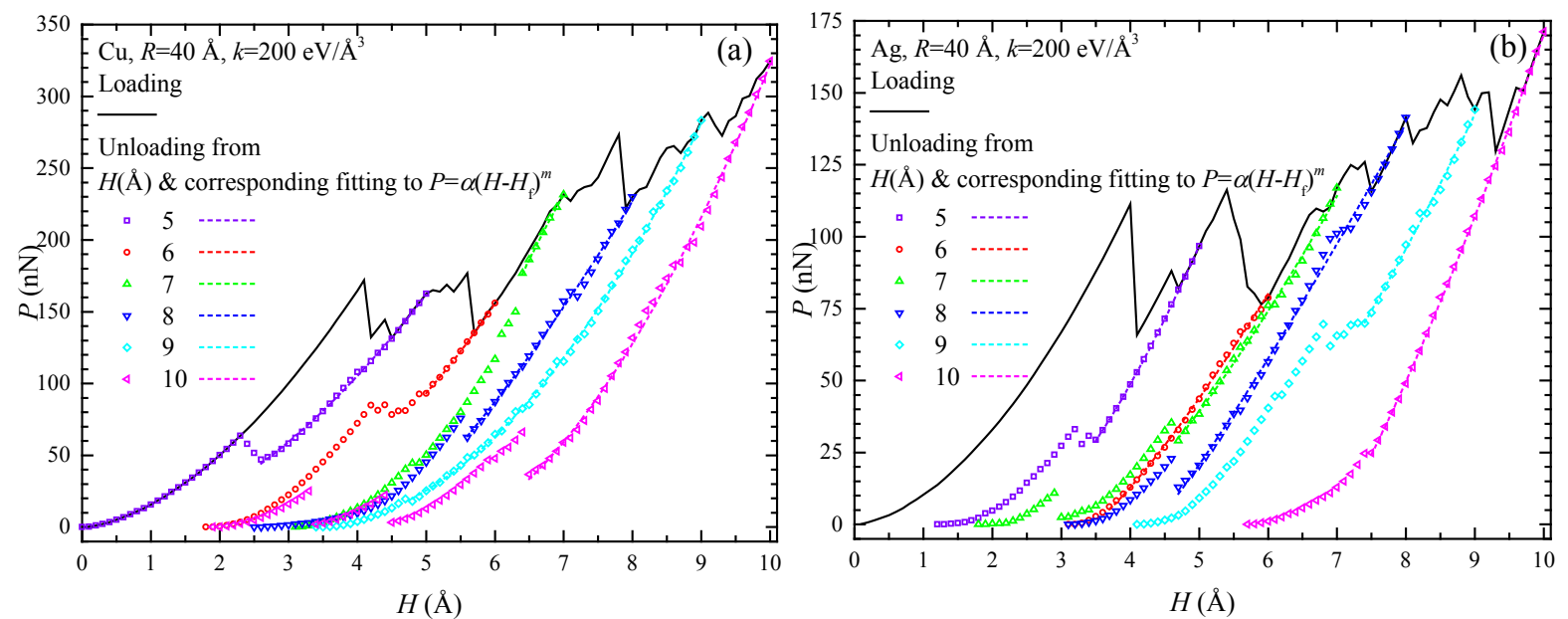

Fig. 8. Load-depth curves for unloading from different depths and their fitting to the power function for nanoindentation on: (a) $\mathrm{Cu}(111)$, (b) $\mathrm{Ag}(111)$. 
After the fitting, $A_{\mathrm{c}}^{\mathrm{O}-\mathrm{P}}$ was easily calculated by Eqs. (11) and (12), and the difference between $H_{\mathrm{IT}}^{\mathrm{O}-\mathrm{P}}$ and $H_{\mathrm{IT}}^{\text {bou }}$ can be characterized by:

$$
\Delta H_{\mathrm{IT}}=\left(H_{\mathrm{IT}}^{\mathrm{O}-\mathrm{P}}-H_{\mathrm{IT}}^{\text {bou }}\right) / H_{\mathrm{IT}}=\frac{P_{\max } / A_{\mathrm{c}}^{\mathrm{O}-\mathrm{P}}-P_{\max } / A_{\mathrm{c}}^{\text {bou }}}{P_{\max } / A_{\mathrm{c}}^{\text {bou }}}=\frac{A_{\mathrm{c}}^{\text {bou }}-A_{\mathrm{c}}^{\mathrm{O}-\mathrm{P}}}{A_{\mathrm{c}}^{\mathrm{O}-\mathrm{P}}}
$$

Fig. 9 shows that the values of $\Delta H_{\text {IT }}$ fall over an interval of $0.12-0.32$ for $\mathrm{Cu}(111), 0.10-0.30$ for $\operatorname{Ag}(111)$, indicating that $H_{\mathrm{IT}}^{\mathrm{O}-\mathrm{P}}$ is much higher than $H_{\mathrm{IT}}^{\text {bou }}$. Therefore, the O-P method significantly underestimates the contact depth in all of the atomistic simulation cases, and is not valid for the shallow nanoindentation at the nanoscale. It agrees with the result of the nanoindentation simulation on a $\mathrm{Cu}(001)$ surface [15]. The reason is that the metal at the nanoscale presents a significant discrete nature of the atomic constitution, and thus can no longer be regarded as a continuum medium.

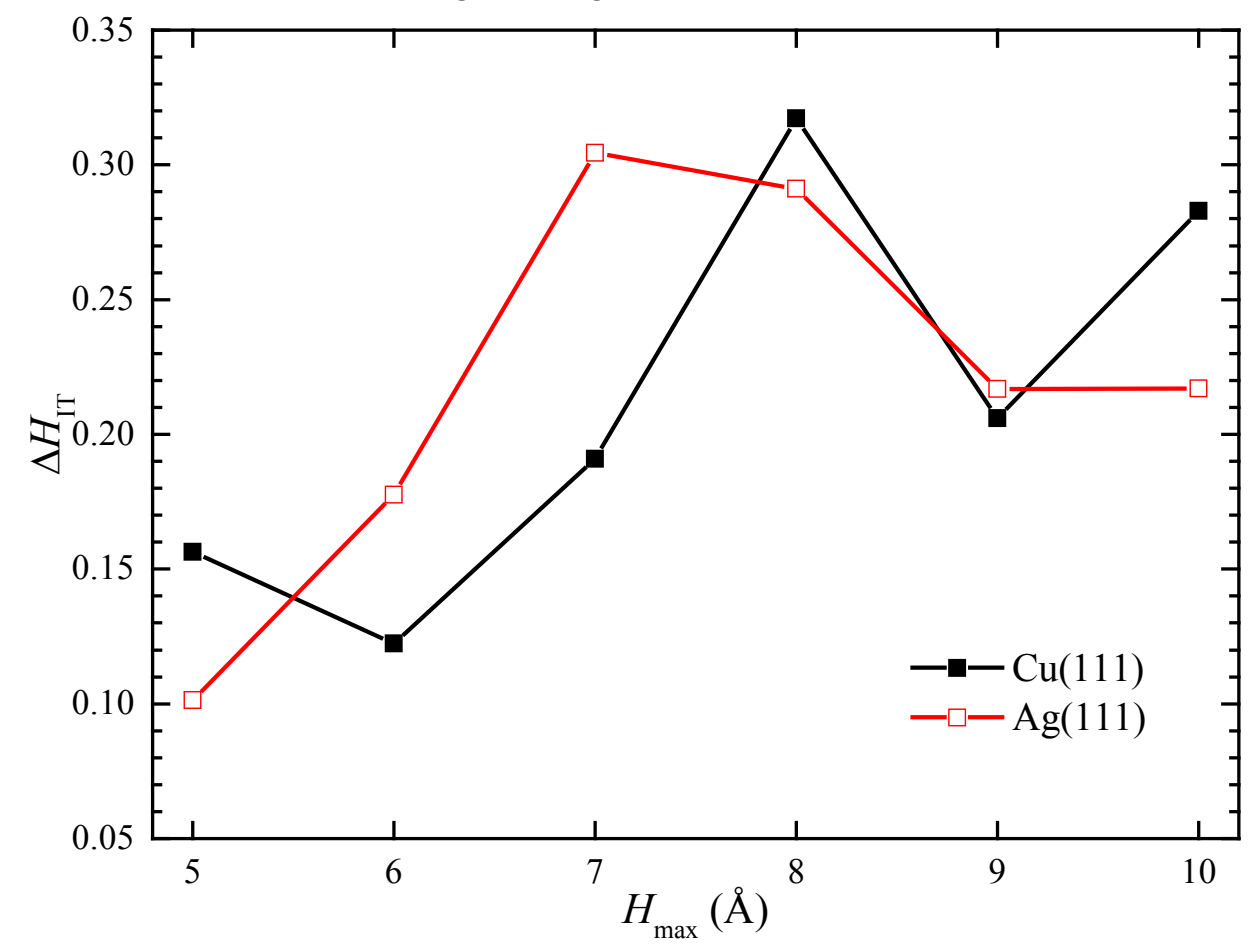

Fig. 9. Comparison of the nanoindentation hardness calculated by two methods at different unloading depths.

\section{Conclusions}

It is crucial to calculate the PCA accurately in order to obtain a reliable value of nanoindentation hardness. In this work, atomistic simulations of nanoindentation were performed on the $\mathrm{Cu}(111)$ and $\operatorname{Ag}(111)$ surfaces, and a new compensational boundary method is proposed to calculate the PCA in atomistic simulations. The key issues of this method include the constitution of the boundary of the projected contact surface, identification of the compensational atom projections and the compensation solution. A simple algorithm based on the Matlab ${ }^{\circledR}$ functions is given as an example of realization. Compared with other available methods, this method provides a clear physical implication, and works well independently of the contact depth and the deformation behaviour of the material. It is also concluded that the O-P method, based on the continuum contact model and widely adopted in experiments, significantly underestimates the PCA in atomistic simulations, and does not work for shallow nanoindentation at the nanoscale.

\section{Acknowledgements}

This work was supported by grants from the China Postdoctoral Science Foundation (Grant No. 2013M540277), Heilongjiang Postdoctoral Fund (Grant No. LBH-Z13089), National Natural Science 
Foundation of China (Grant No. 51175110), and Natural Scientific Research Innovation Foundation in Harbin Institute of Technology (HIT.NSRIF.2015006).

\section{References}

[1] H. Nili, K. Kalantar-zadeh, M. Bhaskaran, S. Sriram, In situ nanoindentation: Probing nanoscale multifunctionality, Prog. Mater. Sci. 58 (2013) 1-29.

[2] M.L.B. Palacio, B. Bhushan, Depth-sensing indentation of nanomaterials and nanostructures, Mater. Charact. 78 (2013) 1-20.

[3] I. Szlufarska, Atomistic simulations of nanoindentation, Mater. Today 9 (2006) 42-50.

[4] A. Ukwatta, A. Achuthan, A molecular dynamics (MD) simulation study to investigate the role of existing dislocations on the incipient plasticity under nanoindentation, Comput. Mater. Sci. 91 (2014) 329-338.

[5] W.C. Oliver, G.M. Pharr, Measurement of hardness and elastic modulus by instrumented indentation: Advances in understanding and refinements to methodology, J. Mater. Res. 19 (2004) 3-20.

[6] W.C. Oliver, G.M. Pharr, An improved technique for determining hardness and elastic modulus using load and displacement sensing indentation experiments, J. Mater. Res. 7 (1992) 1564-1583.

[7] A.C. Barone, M. Salerno, N. Patra, D. Gastaldi, E. Bertarelli, D. Carnelli, P. Vena, Calibration issues for nanoindentation experiments: direct atomic force microscopy measurements and indirect methods, Microsc. Res. Tech. 73 (2010) 996-1004.

[8] F. Sansoz, T. Gang, A force-matching method for quantitative hardness measurements by atomic force microscopy with diamond-tipped sapphire cantilevers, Ultramicroscopy 111 (2010) 11-19.

[9] F. Chen, R. Chang, Study of the effect of imperfect tips on nanoindentation by FEM, J. Mech. Sci. Technol. 21 (2007) 1471-1476.

[10] M.S. Kashani, V. Madhavan, Analysis and correction of the effect of sample tilt on results of nanoindentation, Acta Mater. 59 (2011) 883-895.

[11] J.A. Zimmerman, C.L. Kelchner, P.A. Klein, J.C. Hamilton, S.M. Foiles, Surface step effects on nanoindentation, Phys. Rev. Lett. 87 (2001) 165507.

[12] D. Beegan, S. Chowdhury, M.T. Laugier, Work of indentation methods for determining copper film hardness, Surf. Coat. Technol. 192 (2005) 57-63.

[13] J.K. Deuschle, H. Matthias Deuschle, S. Enders, E. Arzt, Contact area determination in indentation testing of elastomers, J. Mater. Res. 24 (2009) 736-748.

[14] S.P. Ju, C.T. Wang, C.H. Chien, J.C. Huang, S.R. Jian, The nanoindentation responses of nickel surfaces with different crystal orientations, Mol. Simul. 33 (2007) 905-917.

[15] Y.R. Jeng, C.M. Tan, Investigation into the nanoindentation size effect using static atomistic simulations, Appl. Phys. Lett. 89 (2006) 251901.

[16] J.D. Kiely, J.E. Houston, Nanomechanical properties of Au (111), (001), and (110) surfaces, Phys. Rev. B 57 (1998) 12588-12594.

[17] T. Tsuru, Y. Shibutani, Anisotropic effects in elastic and incipient plastic deformation under (001), (110), and (111) nanoindentation of Al and Cu, Phys. Rev. B 75 (2007) 035415.

[18] S. Chen, F. Ke, MD simulation of the effect of contact area and tip radius on nanoindentation, Sci. China Ser. G: Phys. Mech. Astron. 47 (2004) 101-112.

[19] A.V. Verkhovtsev, A.V. Yakubovich, G.B. Sushko, M. Hanauske, A.V. Solov'yov, Molecular dynamics simulations of the nanoindentation process of titanium crystal, Comput. Mater. Sci. 76 (2013) 20-26.

[20] K.J. Van Vliet, J. Li, T. Zhu, S. Yip, S. Suresh, Quantifying the early stages of plasticity through nanoscale experiments and simulations, Phys. Rev. B 67 (2003) 104105.

[21] E.T. Lilleodden, J.A. Zimmerman, S.M. Foiles, W.D. Nix, Atomistic simulations of elastic deformation and dislocation nucleation during nanoindentation, J. Mech. Phys. Solids 51 (2003) 901-920. 
[22] Y. Mishin, M.J. Mehl, D.A. Papaconstantopoulos, A.F. Voter, J.D. Kress, Structural stability and lattice defects in copper: ab initio, tight-binding, and embedded-atom calculations, Phys. Rev. B 63 (2001) 224106.

[23] P.L. Williams, Y. Mishin, J.C. Hamilton, An embedded-atom potential for the $\mathrm{Cu}-\mathrm{Ag}$ system, Model. Simul. Mater. Sci. Eng. 14 (2006) 817-833.

[24] S.J. Plimpton, Fast parallel algorithms for short-range molecular dynamics, J. Comput. Phys. 117 (1995) 1-19.

[25] D. Du, F. Hwang, Computing in Euclidean Geometry, second ed., World Scientific Publishing Co. Pte. Ltd., Singapore, 1995. 